\title{
Event-triggered Output Feedback Control for Uncertain Systems
}

\author{
Zhiwen Wang ${ }^{1,2, a}$, Ying Zhao ${ }^{1, b}$, Chaoqun Zhu ${ }^{1, c}$, Xuan $\mathrm{Li}^{1, \mathrm{~d}}$ \\ ${ }^{1}$ College of Electrical and Information Engineering, Lanzhou University of Technology, Lanzhou, \\ 730050, China \\ ${ }^{2}$ College of Control Science and Engineering, Dalian University of Technology, Dalian, 116026, \\ China \\ aemail:wwwangzhiwen@163.com, bemail:z901220y@163.com, ‘email:chaoqunzhu@yeah.net, \\ demail:Illlixuan@163.com
}

Keywords: Networked Control Systems; Event-triggered Control; Output Feedback; Observer

\begin{abstract}
In this paper, the event-triggered output feedback control problem is studied for uncertain networked control systems. Considering the full states information of system cannot be measured in practice, output feedback control with dynamic compensation function and observer-based state feedback control are derived respectively. Then, novel stability criterions are established to guarantee the asymptotic stability of the closed-loop system based on the theory of Lyapunov stability and linear matrix inequality approach. Numerical examples are given to demonstrate the effectiveness of the proposed method.
\end{abstract}

\section{Introduction}

Networked Control Systems (NCSs) are distributed control system based on a communication network. Compared with the traditional point-to-point control system, NCSs have many advantages such as lower cost, less wiring, higher reliability, information resources sharing. However, control systems faced some issues to be addressed because of the involvement of the communication network, including time delay, packet dropout, communication constraints and so on. In recent years, event-triggered control method has been widespread concerned to solve these problems. The event-triggered control is that sampled signal is transmitted only if the predefined event-triggered condition is violated. In NCSs, the event-triggered control transfers data based on requirement of systems which avoid waiting time for sampling, decrease time delay, improve the utilization of feedback data.

Recently, most event-triggered approaches are based on state feedback control methods [1-4]. However, those methods are inapplicable, because the full states of system cannot be measured, in practice. Therefore, it is absolutely essential to research the event-triggered output feedback control. Event-based output-feedback control method has been proposed in [5]. The stability and the performance of $\mathrm{L}_{\infty}$-gain have been investigated for output-based event-triggered control system [6]. Achieving $\mathrm{L}_{2}$ stability in the presence of communication delays and signal quantization based on passivity theory for event-triggered output feedback control systems has been shown in [7]. The new sufficient condition of an output-based discrete event-triggered mechanism has been introduced in [8]. By using event-triggering quantisation, the hybrid system model and the time delay model has been developed in dynamic output feedback control for linear systems [9]. Inspired by these papers, this paper focuses on event-triggered output feedback control for uncertain networked control systems.

This paper is organized as follows. First, output feedback control model and observer-based state feedback control model have been constructed and analyzed. Then, the new sufficient conditions have been presented in order to insure the asymptotic stability of the closed-loop system. Finally, simulations are shown to prove the effectiveness of the methods.

\section{Problem Description}

Consider the continuous-time linear system with uncertain 
$\left\{\begin{array}{l}\dot{x}(t)=(A+\Delta A) x(t)+B u(t) \\ y(t)=C x(t)\end{array}\right.$

where $x(t) \in R^{n}, u(t) \in R^{m}$ and $y(t) \in R^{r}$ are, respectively, the system state vector, input vector and output vector. all $\Delta A$ satisfying $\Delta A=D F(t) E, A, B, C, D$ and $E$ are known real matrices with appropriate dimensions, $F(t)$ is an unknown matrix to meet $F(t)^{T} F(t) \leq I$.

In the following networked control systems, event generator will be used to determine that whether the sampled signal should be transmitted or not. The sampled signal is transmitted only when the predefined event-triggered condition is violated. In this paper, event-triggered condition depends on the error between the current sampling output date and the recent sampling update output date for transmission, and the current sampling output date. $\left\{t_{k} \mid k \in N\right\}$ is defined as the gather of moments that the event generator transfers sampling update output date.

Event-triggered output-feedback control.

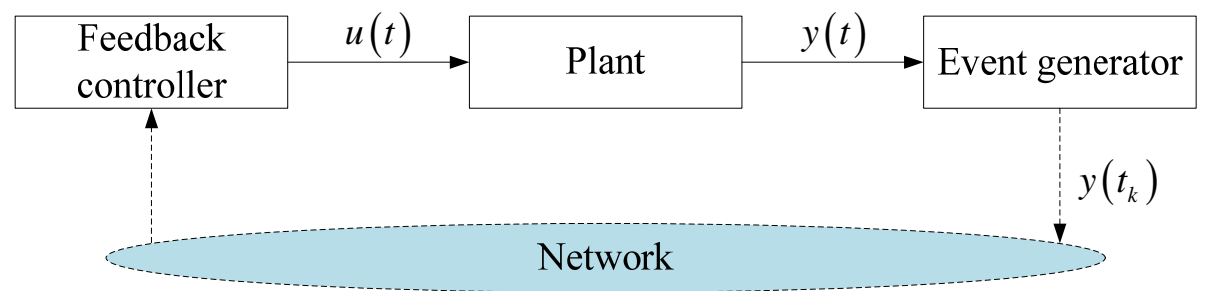

Fig.1. Event-triggered output feedback control loop

The structure of event-triggered output feedback control loop as considered in this paper is shown in Figure 1. Output variable $y(t)$ starts update only if the predefined event-triggered condition is violated. $t_{k}, k \in N$ is used to denote the moment of date updating. Consider the output feedback control law

$u(t)=K y\left(t_{k}\right), t \in\left[t_{k}, t_{k+1}\right), k \in N$

with $K$ is output feedback control gain. Output error of system is

$e_{y}(t)=y\left(t_{k}\right)-y(t), t \in\left[t_{k}, t_{k+1}\right), k \in N$

Therefore, the event-triggered condition is

$\left\|e_{y}(t)\right\|^{2} \leq \sigma\|y(t)\|^{2}, \sigma>0, t \in R^{+}$

When event-triggered condition (4) is violated, event generator is triggered, and control signal is transmitted.

And then, the close-loop system (1) can be expressed as follows

$\left\{\begin{array}{l}\dot{x}(t)=(A+\Delta A) x(t)+B K C x(t)+B K e_{y}(t) \\ y(t)=C x(t)\end{array}\right.$

Event-triggered observer-based state feedback control.

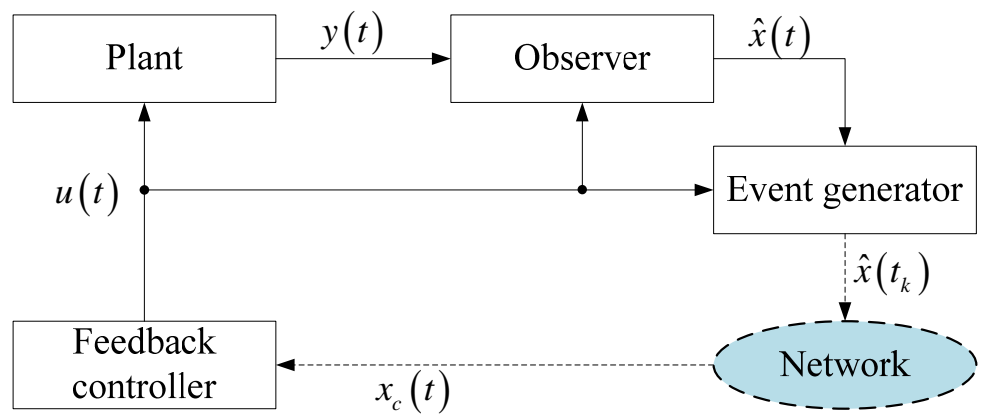

Fig.2. Event-triggered observer-based state feedback control loop

The architecture of event-triggered observer-based state feedback control loop is presented in 
Figure 2. State estimation variable $\hat{x}(t)$ proceeds transfer only if the predefined event-triggered condition is violated. $t_{k}, k \in N$ is defined as the moment of date transmission, and there exists $x_{c}\left(t_{k}\right)=\hat{x}\left(t_{k}\right)$.

The full dimension state observer is given by

$\left\{\begin{array}{l}\dot{\hat{x}}(t)=A \hat{x}(t)+B u(t)+L(y(t)-\hat{y}(t)) \\ \hat{y}(t)=C \hat{x}(t)\end{array}\right.$

with $\hat{x}(t) \in R^{n}$ and $\hat{y}(t) \in R^{r}$ are state vector of observer and output vector of observer, respectively, and $L$ is observer gain matrix.

The model of controller gives

$\left\{\begin{array}{l}\dot{x}_{c}(t)=A x_{c}(t)+B u(t) \\ y_{c}(t)=C x_{c}(t)\end{array}\right.$

We assume that state feedback controller is

$u(t)=K x_{c}(t)$

where $K$ is state feedback control gain. $e(t)=x(t)-\hat{x}(t)$ is used to define the observation error of system, so there is the equation of observation error

$\dot{e}(t)=(A-L C) \tilde{x}(t)+\Delta A x(t)$

The output error of system is defined as $\bar{e}_{y}(t)=y(t)-y_{c}(t)$. In the similar way, the event-triggered condition is given by

$\left\|\bar{e}_{y}(t)\right\|^{2} \leq \delta\|y(t)\|^{2}, \delta>0, t \in R^{+}$

When event-triggered condition (10) is violated, event generator is triggered, and control signal is transmitted.

Based on the description above, the error between the state variable $x(t)$ of plant and the input variable $x_{c}(t)$ of controller is $\tilde{x}(t)=x(t)-x_{c}(t)$, it equals to $\tilde{x}(t)=x(t)-\hat{x}(t)+\hat{x}(t)-x_{c}(t)$, then there is

$$
\dot{\tilde{x}}(t)=(A-L C) \tilde{x}(t)+\Delta A x(t)+\bar{e}_{y}(t)
$$

Therefore, the close-loop system (1) can be expressed as follows

$$
\left\{\begin{array}{l}
\dot{x}(t)=(A+\Delta A+B K) x(t)-B K \tilde{x}(t) \\
y(t)=C x(t)
\end{array}\right.
$$

\section{Main Results} result.

We first introduce the following three lemmas, which are important for developing the main

Lemma 1: Consider matrices $X$ and $Y$ with appropriate dimensions, the following inequality is established:

$$
X^{T} Y+X Y^{T} \leq X^{T} X+Y Y^{T}
$$

Lemma 2: (Schur complement lemma) Consider a symmetric matrix

$$
S=\left[\begin{array}{ll}
S_{11} & S_{12} \\
S_{21} & S_{12}
\end{array}\right]
$$

the following three conditions are equivalent:
(i) $S<0$;
(ii) $S_{11}<0, S_{22}-S_{12}{ }^{T} S_{11}{ }^{-1} S_{12}<0$; 
(iii) $S_{22}<0, S_{11}-S_{12} S_{22}{ }^{-1} S_{12}{ }^{T}<0$.

Lemma 3: Assume that $Q, D$ and $Y$ are real matrices of appropriate dimensions, where $Q$ is a symmetric matrix, then

$$
Q+D F(t) E+E^{T} F(t)^{T} D^{T}<0
$$

holds for all $F(t)$ satisfying $F(t)^{T} F(t) \leq I$, if and only if there exist a constant $\varepsilon>0$, $Q+\varepsilon D D^{T}+\varepsilon^{-1} E^{T} E<0$.

Theorem 1: Analysis the closed-loop system (5), for given output feedback gain $K$, if there exist a symmetric matrix $P \in R^{n \times n}$, scalar $\sigma>0$ and $\varepsilon>0$ that meets following linear matrix inequality :

$$
\left[\begin{array}{ccccc}
P A+A^{T} P+P B K C+C^{T} K^{T} B^{T} P & P B K & C^{T} & P D & E^{T} \\
* & -I & 0 & 0 & 0 \\
* & * & -\sigma^{-1} & 0 & 0 \\
* & * & * & -\varepsilon^{-1} & 0 \\
* & * & * & * & -\varepsilon
\end{array}\right]<0
$$

with known matrices of appropriate dimensions $A, B, C, D$ and $E$, therefore we can prove asymptotic stability of system (5).

Proof: Construct the following Lyapunov function as

$$
V(x)=x(t)^{T} P x(t)
$$

with $P$ is a symmetric matrix. Taking the time derivative of $V(x)$ along the trajectory of system (5) yields

$$
\begin{aligned}
& \dot{V}(x)=\dot{x}(t)^{T} P x(t)+x(t)^{T} P \dot{x}(t) \\
= & x(t)^{T}(A+D F(t) E+B K C)^{T} P x(t)+e_{y}(t)^{T}(B K)^{T} P x(t)+x(t)^{T} P(A+D F(t) E+B K C) x(t) \\
& +x(t)^{T} P B K e_{y}(t)
\end{aligned}
$$

Then based on the lemma 1 and event-triggered condition (4), the following equation can be obtained.

$$
\begin{aligned}
& \dot{V}(x) \leq x(t)^{T}\left[P(A+D F(t) E+B K C)+(A+D F(t) E+B K C)^{T} P\right] x(t)+x(t)^{T} P B K K^{T} B^{T} P x(t) \\
& +e_{y}(t)^{T} e_{y}(t) \\
& \leq x(t)^{T}\left[P(A+D F(t) E+B K C)+(A+D F(t) E+B K C)^{T} P+P B K K^{T} B^{T} P\right] x(t)+\sigma y(t)^{T} y(t) \\
= & x(t)^{T}\left[P(A+D F(t) E+B K C)+(A+D F(t) E+B K C)^{T} P+P B K K^{T} B^{T} P+\sigma C^{T} C\right] x(t) \\
& =x(t)^{T} \Psi x(t)
\end{aligned}
$$

Obviously, system (5) is asymptotic stability when $\Psi<0$. According to lemma 3$$
\left[\begin{array}{cc}
P A+A^{T} P+P B K C+C^{T} K^{T} B^{T} P+\varepsilon P D D^{T} P+\varepsilon^{-1} E^{T} E+P B K K^{T} B^{T} P & C^{T} \\
* & -\sigma^{-1}
\end{array}\right]<0
$$

Finally, we can reach linear matrix inequality (13) via lemma 2.

Theorem 2: Analysis the closed-loop system (12), for given feedback gain $K$ and observer gain $L$, if there exist a symmetric matrix $Q \in R^{n \times n}$ and $R \in R^{n \times n}$, scalar $\sigma>0$ and $\varepsilon>0$ that meets following linear matrix inequality : 


$$
\left[\begin{array}{ccccccc}
(A+B K)^{T} Q+Q(A+B K) & -Q B K & 0 & C^{T} & Q D & E^{T} & \Delta A^{T} \\
* & R(A-L C)+(A-L C)^{T} R & R & 0 & 0 & 0 & 0 \\
* & * & -2 I & 0 & 0 & 0 & 0 \\
* & * & * & -\delta^{-1} & 0 & 0 & 0 \\
* & * & * & * & -\varepsilon^{-1} & 0 & 0 \\
* & * & * & * & * & -\varepsilon & 0 \\
* & * & * & * & * & * & -I
\end{array}\right]<0(15)
$$

with $A, B, C, D$ and $E$ are known matrices with appropriate dimensions, hence the asymptotic stability of system (12) can be tested.

Proof: Construct the following Lyapunov function as

$$
V[x(t), \tilde{x}(t)]=x(t)^{T} Q x(t)+\tilde{x}(t)^{T} R \tilde{x}(t)
$$

with $P$ and $Q$ are symmetric matrices. The time derivative of $V[x(t), \tilde{x}(t)]$ along the state trajectory of system (12) and error equation (13) are

$$
\begin{aligned}
& \dot{V}[x(t), \tilde{x}(t)]=\dot{x}(t)^{T} Q x(t)+x(t)^{T} Q \dot{x}(t)+\dot{\tilde{x}}(t)^{T} R \tilde{x}(t)+\tilde{x}(t)^{T} R \dot{\tilde{x}}(t) \\
& =x(t)^{T}(A+\Delta A+B K)^{T} Q x(t)-\tilde{x}(t)^{T} K^{T} B^{T} Q x(t)+x(t)^{T} Q(A+\Delta A+B K) x(t)-x(t)^{T} Q B K \tilde{x}(t) \\
& \quad+\tilde{x}(t)^{T}(A-L C)^{T} R \tilde{x}(t)+x(t)^{T} \Delta A^{T} R \tilde{x}(t)+\bar{e}_{y}(t)^{T} R \tilde{x}(t)+\tilde{x}(t)^{T} R(A-L C) \tilde{x}(t) \\
& \quad+\tilde{x}(t)^{T} R \Delta A x(t)+\tilde{x}(t)^{T} R \bar{e}_{y}(t)
\end{aligned}
$$

Then lemma 1 and the event-triggered condition given in (10) will be applied in the following equation.

$$
\begin{aligned}
& \dot{V}[x(t), \tilde{x}(t)] \leq x(t)^{T}\left[(A+\Delta A+B K)^{T} Q+Q(A+\Delta A+B K)+\Delta A^{T} \Delta A\right] x(t) \\
& \quad+\tilde{x}(t)^{T}\left[R(A-L C)+(A-L C)^{T} R+2 R R\right] \tilde{x}(t)-\tilde{x}(t)^{T} K^{T} B^{T} Q x(t)-x(t)^{T} Q B K \tilde{x}(t)+\bar{e}_{y}(t)^{T} \bar{e}_{y}(t) \\
& \leq x(t)^{T}\left[(A+\Delta A+B K)^{T} Q+Q(A+\Delta A+B K)+\Delta A^{T} \Delta A\right] x(t)+\tilde{x}(t)^{T}\left[R(A-L C)+(A-L C)^{T} R+2 R R\right] \tilde{x}(t) \\
& \quad-\tilde{x}(t)^{T} K^{T} B^{T} Q x(t)-x(t)^{T} Q B K \tilde{x}(t)+\delta y(t)^{T} y(t) \\
& =\left[\begin{array}{c}
x(t) \\
\tilde{x}(t)
\end{array}\right]^{T}\left[\begin{array}{cr}
(A+\Delta A+B K)^{T} Q+Q(A+\Delta A+B K)+\Delta A^{T} \Delta A+\delta C^{T} C & -Q B K \\
* & R(A-L C)+(A-L C)^{T} R+2 R R
\end{array}\right]\left[\begin{array}{l}
x(t) \\
\tilde{x}(t)
\end{array}\right] \\
& =\left[\begin{array}{c}
x(t) \\
\tilde{x}(t)
\end{array}\right]^{T} \Phi\left[\begin{array}{l}
x(t) \\
\tilde{x}(t)
\end{array}\right]
\end{aligned}
$$

It is obvious that when $\Phi<0$ the close-loop system (12) is asymptotic stability. According to lemma 3

$$
\left[\begin{array}{cc}
(A+B K)^{T} Q+Q(A+B K)+\varepsilon Q D D^{T} Q+\varepsilon^{-1} E^{T} E+\Delta A^{T} \Delta A+\delta C^{T} C & -Q B K \\
* & R(A-L C)+(A-L C)^{T} R+2 R R
\end{array}\right]<0
$$

At last, linear matrix inequality (15) is obtained through using lemma 2.

\section{Simulation Examples}

In order to illustrate the effectiveness of the proposed method, Let us consider the continuous-time linear system with uncertain, whose matrices in (1) are given by

$$
A=\left[\begin{array}{cc}
0 & 1 \\
-1 & -2
\end{array}\right], B=\left[\begin{array}{l}
0 \\
1
\end{array}\right], C=\left[\begin{array}{ll}
0 & 2 \\
1 & 0
\end{array}\right], D=\left[\begin{array}{ll}
1 & 0 \\
3 & 5
\end{array}\right], E=\left[\begin{array}{ll}
3 & 1 \\
2 & 4
\end{array}\right]
$$


By verifying that system is controllable and observable, the matrix of feedback gain $K$ and the matrix of observer gain $L$ are calculated by the method of pole assignment.

$$
K=\left[\begin{array}{ll}
4 & 2.5
\end{array}\right], L=\left[\begin{array}{cc}
0.5 & 2.25 \\
6 & -1
\end{array}\right]
$$

According to the theorem 1, we invoke the feasibility problem solver feasp function from LMI Toolbox and obtain $\operatorname{tmin}=-0.0606<0$. The result can prove that this close-loop system exists a group of feasible solutions:

$$
P=\left[\begin{array}{cc}
0.7338 & -0.1242 \\
-0.1242 & -0.2009
\end{array}\right], \sigma=49.2192, \varepsilon=40.7729
$$

In the same way, we consider the theorem 2 , and then obtain $\operatorname{tmin}=-0.9687<0$, so a group of feasible solutions is

$$
Q=\left[\begin{array}{cc}
1.5977 & -2.5190 \\
-2.5190 & -0.0232
\end{array}\right], R=\left[\begin{array}{cc}
-3.4040 & -0.2726 \\
-0.2726 & -1.3509
\end{array}\right], \delta=35.5070, \varepsilon=40.0280
$$

We suppose the initial state of close-loop system is $x_{0}=\left[\begin{array}{ll}2 & 0\end{array}\right]^{T}$, then test the proposal through Matlab simulation. The state responses of system are shown in Figure 3. The availability of event-triggered output feedback control method and observer-based state feedback control method show in Figure 3 (a) and (b).

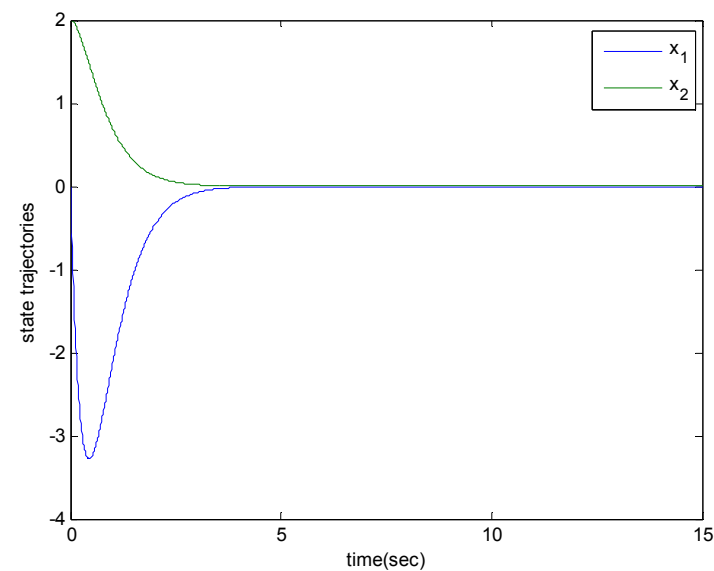

(a) output-feedback control

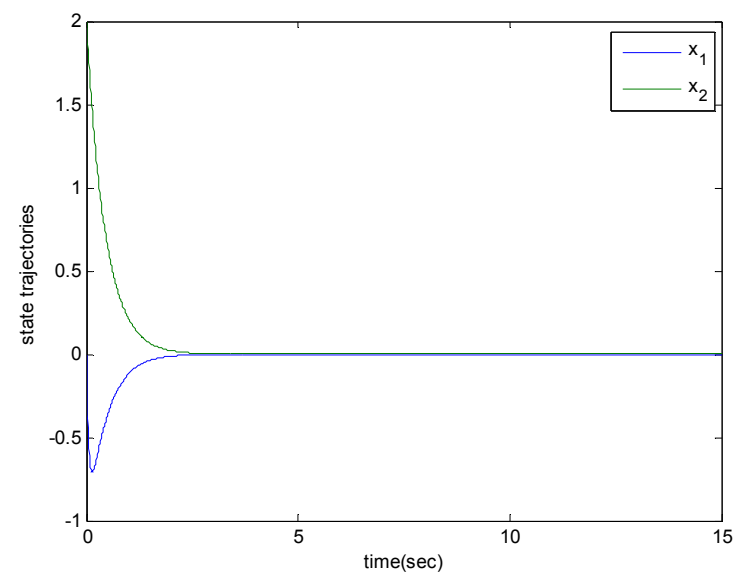

(b) observer-based state feedback control

Fig.3. State responses

\section{Conclusions}

This paper has addressed the event-triggered output feedback control for uncertain networked control systems. Output feedback control method and observer-based state feedback control method have been presented. According to the event-triggered mechanism, two models of closed-loop systems have been setted up. By using Lyapunov function and linear matrix inequality approach, some new sufficient conditions that can guarantee the asymptotic stability of the closed-loop system have been established. At last, two numerical examples have been given to illustrate the feasibility and efficiency.

\section{Acknowledgement}

This work is supported by the National Natural Science Foundation of China (NSFC) under Grant 61263003 and 61563031, the Natural Science Foundation of Gansu Province of China under Grant 148RJZA009, the Fundamental Research Funds for the Central Universities of China under Grant 2009JC11 and 2009QN120, the Doctor Foundation of Lanzhou University of Technology under Grant BS03200901, the Excellent Young Teachers Foundation of Lanzhou University of 
Technology under Grant Q201012.

\section{References}

[1] Daniel Lehmann, Jan Lunze. event-based control: A state-Feedback Approach [C]. Proceedings of the European Control Conference, 2009.1716-1721.

[2] Peng Chen, Chengyang Tai. Event-triggered communication and $H_{\infty}$ control co-design for networked control systems [J]. Automatica, 201249 1326-1332.

[3] W.P.M.H. Heemels, M.C.F. Donkers, A.R. Teel. Periodic event-triggered Control Based on State Feedback [C]. IEEE Conference on Decision and Control and European Control Conference, 2011.2571-2576.

[4] Joao Almeida, Carlos Silvestre, Antonio M. Pascoal. Self-triggered state feedback control of linear plants under bounded disturbances [C]. IEEE Conference on Decision and Control, 2010.7588-7593.

[5] Daniel Lehmann, Jan Lunze. Event-based output-feedback control [C]. IEEE Mediterranean Conference on Control and Automation, 2011.982-987.

[6] M.C.F. Donkers, W.P.M.H. Heemels. Output-based event-triggered control with guaranteed L $\infty$-gain and improved and decentralised event-triggering [J]. IEEE Transactions on Automatic Control, 2012 57(6) 1362-1376.

[7] Han Yu, Panos J. Antsaklis. Event-triggered output feedback control for networked control systems using passivity: Achieving $L_{2}$ stability in the presence of communication delays and signal quantization [J]. Automatica, 201349 30-38.

[8] Xiaoming Zhang, Qinglong Han. Event-based dynamic output feedback control for networked control systems [J]. IET Control Theory and Applications, 2014 8(4) 226-234.

[9] Ce Liu, Fei Hao. Dynamic output-feedback control for linear systems by using event-triggered quantization [J]. IET Control Theory and Applications, 2015 9(8) 1254-1263. 